\title{
Le district de santé d'Akonolinga
}

\section{Raphaël Okalla}

\section{(2) OpenEdition}

Journals

Édition électronique

URL : http://journals.openedition.org/apad/33

DOI : 10.4000/apad.33

ISSN : 1950-6929

Éditeur

LIT Verlag

\section{Édition imprimée}

Date de publication : 1 juin 2001

Référence électronique

Raphaël Okalla, «Le district de santé d'Akonolinga », Bulletin de l'APAD [En ligne], 21 | 2001, mis en ligne le 06 décembre 2005, consulté le 07 septembre 2020. URL : http://journals.openedition.org/apad/33 ; DOI : https://doi.org/10.4000/apad.33

Ce document a été généré automatiquement le 7 septembre 2020

Bulletin de l'APAD 


\title{
Le district de santé d'Akonolinga
}

\author{
Raphaël Okalla
}

1 Le département du Nyong et Mfoumou, dans la province du Centre, est divisé en trois arrondissements : Akonolinga, Endom et Ayos. En accord avec le délégué provincial de la santé de la Province du Centre, chaque arrondissement a d'abord fonctionné au départ comme un district de santé, sous la coordination du service départemental de la santé. A la suite de la réunion de Bertoua sur la délimitation des districts, il est finalement décidé que les arrondissements d'Endom et d'Akonolinga formeront un seul et même district de santé dénommé "district d'Akonolinga", l'arrondissement d'Ayos entrant dans un district indépendant. Cependant Endom ne sera intégré de manière opérationnelle à Akonolinga qu'à partir de 1994. Une réorganisation administrative du territoire après 1994 a créé un district administratif à l'intérieur de l'arrondissement d'Akonolinga : le district de Mengang. Cette division n'a pas modifié la configuration district.

2 Le district de santé d'Akonolinga couvre une superficie de $4.300 \mathrm{Km}^{2}$ et compte 77.539 habitants en 1998. Il couvre trois unités administratives: l'arrondissement d'Akonolinga, l'arrondissement d'Endom et le district (administratif) de Mengang. La population est en majorité rurale; trois agglomérations concentrent quelques fonctionnaires, des employés de quelques entreprises privées, et attirent les jeunes des villages à la recherche d'emplois, des avantages de la ville (électricité, musique, cinémas, bars), et fuyant la sorcellerie du village. Cette région, est essentiellement agricole et forestière. Des planteurs produisent cacao et café; et depuis quelques années, les cultivatrices vendent à certains pays voisins de la sous région (Gabon et Guinée Equatoriale) certains produits vivriers comme le taro, le plantain, les arachides. L'élevage et la pêche se pratiquent de manière artisanale et leur production entre dans la consommation locale. Plusieurs entreprises effectuent la coupe des grumes ; certaines commencent à implanter des scieries sur place pour la transformation du bois et créent ainsi quelques emplois. Akonolinga est située à $135 \mathrm{Km}$ de Yaoundé qui est accessible par une route bitumée. Cependant le réseau routier à l'intérieur du district est très dégradé. Certains centres de santé sont inaccessibles pendant la saison des pluies, et il faut parfois plus de quatre heures avec un véhicule tout terrain pour 
parcourir les $70 \mathrm{~km}$ qui séparent le centre de santé d'Akak au service de santé de district installé à Akonolinga.

Le Projet santé Nyong et Mfoumou

3 Le district de santé a bénéficié de l'appui du "Projet santé Nyong et Mfoumou", conçu comme un projet de développement global concernant les secteurs de la santé, de l'agriculture et de l'éducation nationale, associant le Ministère de la santé publique, la société de développement du cacao du Nyong et Mfoumou et l'Association Française des Volontaires du Progrès (AFVP). Le projet porté par l'AFVP consistait à créer des centres de santé qui fonctionnent selon les prescriptions du document de la Réorientation des soins de santé primaires et à préparer le département à la future organisation du pays en districts de santé. Le projet avait pour but la rationalisation de 9 centres de santé du district d'Akonolinga. Il octroyait à chaque centre une moto, un stock initial de médicaments et du matériel. L'AFVP appuyait les activités de suivi et de contrôle avec des ressources logistiques conséquentes (véhicule, carburant), il a également mis en place un dépôt départemental de médicaments.

4 Les autorités administratives, sanitaires et traditionnelles, les assistants techniques de l'AFVP, et les élus locaux ont procédé aux délimitations des aires de santé au début du projet en 1990. Le nombre de centres de santé à construire et à rénover a été fixé à neuf, en commençant par trois centres. Il fallait en effet concentrer les capacités de gestion et d'organisation sur un nombre réduit de centres, ce qui permettrait également d'acquérir de l'expérience. Au début du projet, la REOSSP n'est pas encore opérationnelle, et le texte qui servait de base, élaboré par la Direction de la médecine préventive, donnait les grandes orientations, mais pas les détails pratiques. L'équipe du projet était composée de trois assistants techniques de l'AFVP, des trois médecins chefs de service de santé d'arrondissements (Akonolinga, Ayos, Endom), et du chef de service départemental de la santé.

5 Le projet a commencé par une information des différents partenaires (autorités administratives, leaders religieux, populations) sur l'organisation qui allait se mettre en place ; cette première phase de sensibilisation fut rapidement exécutée, en moyenne deux à trois heures étaient consacrées à chaque village. Après la sensibilisation, chaque village devait élire deux représentants; l'élection se déroulait sous la supervision d'un membre de l'équipe du projet qui ne devait pas repartir du village sans les noms des élus. Après les élections dans chaque village de l'aire de santé, le chef de service de santé d'arrondissement réunissait tous les représentants de l'aire de santé pour former le comité de santé et en élire le bureau et procéder à l'élection des membres du comité de gestion. Le recensement commençait après cette étape, les membres du comité de santé devaient participer à ce travail et faciliter les contacts avec les communautés. Chaque représentant de la communauté disposait d'un cahier de recensement, et chaque chef de famille devait payer 200 FCFA pour marquer son adhésion au projet; il fallait alors prouver aux bailleurs de fonds de l'AFVP que la communauté participait au financement du projet. Un assistant technique du projet aidait le représentant du village à dresser les fiches de chaque famille où étaient mentionnés le nom du chef de famille et de tous les membres de sa famille. Puis il s'est agi de sélectionner les "commis", soit la personne issue de la communauté chargée de la gestion du centre de santé sous le contrôle du comité de santé. Les représentants de la communauté proposaient alors les noms de quelques jeunes qui devaient au moins être en possession du certificat d'études primaires pour concourir, l'assistant technique 
organisait un concours et retenait la personne qui allait être formée comme commis. La formation des membres du comité de santé, y compris le commis, durait deux jours ; puis les membres du comité de gestion (le président, le trésorier et le secrétaire) et le commis bénéficiaient d'une formation spécifique de trois jours supplémentaires sur la gestion de l'argent issu du recouvrement des coûts; enfin le commis poursuivait sa formation pratique pendant un mois soit avec un assistant technique de l'AFVP, soit auprès d'un autre projet où existaient déjà des commis expérimentés.

6 Simultanément, il s'agissait de rénover ou construire des centres de santé. L'AFVP recrutait des artisans locaux pour les constructions et les rénovations, mais aussi pour les meubles et certains équipements. Les médicaments pour constituer le stock initial de la pharmacie des centres ont été achetés en Europe en commande groupée pour les trois centres de santé pilotes. Le même procédé a été utilisé pour la commande du matériel technique. Les personnels qui devaient travailler dans les centres de santé étaient formés pendant une semaine sur l'organisation des services dans le centre; la formation mettait notamment l'accent sur le "circuit du malade" dans le système de santé. Cependant, le ministère de la santé devait préalablement prendre les décisions d'affectation des personnels dans la région, ce qui a retardé le processus. La mise en place des pharmacies, du matériel, des fiches de gestion et de consultations intervenait dès la réception des bâtiments. Pendant une année, chaque centre faisait l'objet d'un suivi mensuel par des membres du Projet.

La carte sanitaire du district de santé d'Akonolinga

7 Le district de santé d'Akonolinga s'est formé en deux temps. De 1990 à 1994, il couvre d'abord l'arrondissement administratif d'Akonolinga et ses limites territoriales correspondent à celles de l'arrondissement dont il porte le nom. Après le séminaire national de délimitation des districts de santé de Bertoua en 1992, le Ministère de la santé publique a décidé de jumeler les districts d'Akonolinga et d'Endom ${ }^{1}$. Le décret présidentiel créant un district administratif à l'intérieur de l'arrondissement d'Akonolinga en 1994 n'a eu que peu d'effet sur l'organisation du district sanitaire. Le district de santé est subdivisé en onze aires de santé, dont quatre appartenaient à l'ancien district d'Endom (Akak, Edjom, Ekoudou, Endom urbain).

Tableau 1 : Les aires de santé du district sanitaire d'Akonolinga

\begin{tabular}{|l|l|}
\hline Aires de santé & population \\
\hline \hline Akak & 2.688 \\
\hline \hline Akonolinga urbain & 20.622 \\
\hline \hline Edjom & 5.516 \\
\hline \hline Ekoudou & 4.200 \\
\hline \hline Endom urbain & 8.116 \\
\hline \hline Evan So'o & 8.872 \\
\hline \hline Djoujoua & 3.600 \\
\hline
\end{tabular}




\begin{tabular}{|l|l|}
\hline Mengang & 5.500 \\
\hline \hline Megueme & 6.511 \\
\hline \hline Yeme - Yeme & 7.427 \\
\hline \hline Zalom & 4.487 \\
\hline \hline Total & 77.539 \\
\hline
\end{tabular}

(Source : document statistique du Service de santé de district)

Plusieurs réunions de concertation (avec les personnels de la santé et la population) et entretiens (avec le préfet, le sous-préfet, les élus locaux, et les responsables religieux) ont précédé la délimitation des aires de santé. Les responsables sanitaires ont informé leurs interlocuteurs des critères (surtout démographique pour les aires de santé : 5 à 10.000 habitants pour une aire de santé) préconisés par le ministère de la santé. Après discussion avec les différents partenaires, les critères qui ont été pris en considération de commun accord et qui ont été utilisés sur l'étendue du district d'Akonolinga actuel sont les suivants : la localisation des formations sanitaires préexistantes (notamment pour l'aire de santé d'Endom), les affinités socioculturelles (par exemple, pour l'aire de santé de Mengang²), et l'accessibilité géographique (pour l'aire de santé d'Akak). Ces délimitations se sont faites sans grands heurts, et deux cas nous paraissent intéressants à présenter pour percevoir les problèmes rencontrés et les enjeux sous tendus par la délimitation des aires de santé.

L'arrondissement d'Endom a pour chef-lieu la ville d'Endom. A la création de cette unité administrative, une rude bataille avait opposé les élites d'Endom village et d'Edjom village à propos de la localisation du chef lieu de l'arrondissement ${ }^{3}$. La population d'Edjom, considérant la taille de leur agglomération, et sa situation géographique centrale au carrefour de deux provinces et de quatre arrondissements, jugeait que leur village méritait d'être le chef-lieu du nouvel arrondissement. Le village d'Endom quant à lui était le village d'origine d'élites importantes dans la vie politique et administrative du pays et parvint à devenir le chef-lieu en donnant son nom à l'arrondissement. Tous les services administratifs et publics s'y sont installés, y compris l'hôpital d'arrondissement.

Lorsque les "Blancs"4 viennent en appui pour créer des centres de santé, il est évident aux élites du village d'Edjom que le premier centre de santé sera créé dans leur village. Il existait déjà un centre de santé catholique fonctionnel situé à un kilomètre d'Edjom ville, et on ne pouvait pas alors ouvrir un centre de santé à proximité d'un autre existant, privé, et qui fonctionnait bien. Cependant la population d'Edjom, peu sensible à la rationalité de la nouvelle carte sanitaire, n'accepta pas la décision de placer le nouveau centre de santé à Endom, et exercèrent des pressions sur les responsables sanitaires et le sous-préfet pour l'infléchir. Un membre du projet AFVP se souvient : "Ils n'écoutaient pas nos explications sur la population minimale pour faire fonctionner un centre de santé, ni sur la concurrence malsaine qui allait naître avec un centre qui les soigne bien depuis longtemps. J'avais l'impression que ce n'était pas le centre de santé pour se soigner qu'ils voulaient mais avoir une institution publique installée à Edjom 


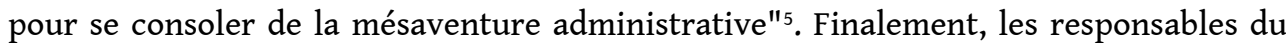
projet et le sous-préfet acceptèrent de créer un centre de santé intégré public à Edjom à un kilomètre du centre de santé catholique fonctionnel.

11 La création de l'aire de santé d'Akak a posé un autre type de problème à l'équipe du projet AFVP. Le village d'Akak est situé à une quarantaine de kilomètres de l'hôpital d'Endom. La route est si mauvaise en saison des pluies qu'il faut trois heures avec un véhicule tout terrain pour l'atteindre à partir d'Endom, et il n'existe pas de transport en commun reliant les deux localités. Le village d'Akak compte à peine 1.500 habitants avec les hameaux voisins. Les responsables du projet ont dû alors adjoindre à l'aire de santé des villages d'un arrondissement voisin, certains villages limitrophes de cette aire de santé étant éloignés de toutes les autres structures de santé existantes, leur centre de santé de référence leur étant inaccessible.

Ces deux exemples montrent les difficultés rencontrées sur le terrain pour appliquer des normes qui ne tenaient compte au départ que des aspects techniques. L'équipe du projet AFVP chargée de la mise en place du district sanitaire dans cette zone en est ainsi arrivée à créer dans un contexte de ressources rares un centre de santé qui n'avait aucune autre justification que des enjeux sociaux et politiques. Par ailleurs, des contraintes liées à l'accessibilité et à la faible densité de certaines régions ont imposé la création d'aires de santé qui ne pourront pas fonctionner sans faire jouer une solidarité dans le district tout entier, car la viabilité économique d'un centre de santé dépend d'un nombre critique d'habitants à partir duquel le recouvrement de coûts peut suffire à la prise en charge des dépenses de fonctionnement d'un centre de santé.

Le district d'Akonolinga compte donc onze centres de santé intégrés publics, trois dispensaires privés confessionnels (deux structures catholiques, Akonolinga et Mebassa ; et une structure protestante, Melan), une formation sanitaire de niveau intermédiaire dénommée centre médical d'arrondissement, et l'hôpital de référence du district. L'hôpital de district a une capacité de 59 lits, et est divisé en quatre services : chirurgie (17 lits), médecine (20 lits), maternité (11 lits), et pédiatrie (11 lits). En 1998, cette formation sanitaire déclare 1.742 consultations, soit une moyenne de 4,7 consultations par jour, ce qui représente une charge de travail de 1,5 consultation par médecin et par jour. L'hôpital utilise trois médecins, un technicien supérieur de soins, trois infirmiers diplômés d'État, six infirmiers brevetés, trois infirmiers brevetés accoucheurs, six techniciens adjoints de laboratoire, onze aides-soignants et sept agents de l'État.

ee centre médical d'arrondissement d'Endom est l'exemple d'un hôpital d'arrondissement construit avant le début de la crise économique. Le bâtiment a été conçu pour abriter un hôpital de 60 lits avec tous les services nécessaires pour un hôpital d'arrondissement. Malheureusement il n'a jamais été équipé en matériel technique standard pour un hôpital de ce type. Le peu de matériel utilisé ici provient des dons et legs. En fait, le centre médical d'arrondissement est aujourd'hui vide de tout (matériel, personnel, médicament), et ne doit plus son nom qu'à la présence d'un médecin qui y est affecté. Il fonctionne à peine comme un centre de santé et bénéficie pourtant des services d'un médecin, de deux infirmiers brevetés et de quatre aidessoignants.

15 Il existe un réseau de tradipraticiens dans la région dont certains semblent faire partie intégrante du système de santé local. Mentionnons l'exemple d'un tradipraticien de l'aire de santé d'Akak qui consultait et "hospitalisait" des malades dans son village, 
avec une infrastructure permettant l'hébergement d'une vingtaine de personnes. Il a été désigné par les habitants du village pour être le Président du comité de santé de son aire de santé. Il entretenait d'excellents rapports avec les services de santé et tenait un registre de ses activités dont les responsables des services de santé étaient régulièrement informés. Le chef de centre se souvient de cette collaboration : "Il exige de tous les malades qui résident chez lui de se présenter pour la vaccination des enfants et des femmes au centre de santé".

La population de l'aire de santé est divisée en deux sous-groupes: une population centrale résidant dans un rayon de cinq kilomètres autour du centre, et au-delà, une population périphérique. L'équipe du centre de santé établit un calendrier de déplacement dans les villages pour des activités préventives et de promotion de la santé (vaccination, consultation prénatale, éducation pour la santé) destinées à la population périphérique, la population centrale étant censée se rendre au centre de santé pour toutes ces activités.

Le secteur privé confessionnel, pour ses centres de santé, a gardé la dénomination de "dispensaire" qui n'a plus cours dans le système actuel ; ce que le service de santé du district lui concède volontiers. Aucune aire de santé du district n'est officiellement couverte par une structure confessionnelle, et le secteur confessionnel n'est pas représenté dans la composition de l'équipe cadre de district. En effet, l'équipe cadre du district, chargée de l'organisation, de la planification et de la mise en œuvre du plan d'action du district est composée du chef de service de santé de district, des trois médecins de l'hôpital de district, du chef du bureau santé, du chef du bureau des affaires administratives et financières, du surveillant général et de l'économe de l'hôpital de district. Pourtant, le secteur confessionnel joue un rôle très important dans les consultations ambulatoires mais intervient très peu dans le fonctionnement du réseau de santé du district. Cependant, le planning des activités du district du second semestre 1999 prévoyait une supervision des centres du district qui concernait aussi les centres confessionnels.

Le parcours du patient

Le malade qui arrive au centre de santé pour une consultation se présente au "commis". Le commis est un membre de la communauté, recruté sur concours, formé pour le recouvrement des coûts pendant un à trois mois dans un centre de santé qui fonctionne bien sur le plan financier ; cette personne est responsable devant le comité de gestion et devant le chef du centre de santé ; il est rémunéré par le comité de gestion à partir des recettes propres du centre provenant du recouvrement des coûts; son salaire dans les centres de cette zone s'élève à 180 FCFA. Le commis délivre un ticket de consultation au patient qui paye alors 100 FCFA. Il se rend ensuite dans le bureau de l'infirmier qui assure la consultation ce jour, puis revient chez le commis auprès duquel il achète les médicaments prescrits; le commis lui explique comment utiliser les remèdes. Dans le cas de traitement difficile à manipuler, le malade retourne voir l'infirmier pour des explications complémentaires. Si le patient est en période d'observation, doit faire l'objet d'injections ou d'un pansement, il se rend dans la salle de soins. Dans le cas des consultations préventives, comme la consultation prénatale, la patiente s'acquitte d'un forfait pour toute la période de sa grossesse; dans ce forfait entrent la prophylaxie du paludisme, la supplémentation en fer, des vitamines ainsi que les consultations mensuelles. Les séances de vaccination, pour les enfants de 0 à 5 ans et les femmes en âge de procréer, sont organisées à jours fixes au centre de santé et sont 
gratuites pour toutes les maladies du programme élargi de vaccination. Mais les centres de santé du district ne proposent pas toutes les activités du paquet minimum aux usagers; par exemple, ils n'organisent pas de consultations préscolaires, et certains centres n'ont pas de service de maternité, se préoccupent peu de planification familiale, ou n'organisent guère la prise en charge des malades chroniques.

Si le centre de santé ne peut pas faire face à un problème de santé, il réfère le patient au centre médical d'arrondissement ou à l'hôpital de district qui traitera son problème ; puis, une fois traité, il est censé être renvoyé dès que possible vers le centre de santé dont il dépend, avec des consignes écrites de surveillance à l'attention du personnel soignant de ce centre. Cela suppose que le plateau technique de l'hôpital de district est supérieur à celui des centres de santé. Dans la pratique, si les malades se présentent effectivement d'abord dans leur centre de santé, le système de référence lui ne fonctionne pas toujours comme prévu.

Le système de référence est perturbé par les très grandes difficultés d'accès à certains centres pendant certaines périodes de l'année. Mais les raisons du non fonctionnement de la référence tient beaucoup plus à des questions d'organisation du système. Les centres de santé ont été construits ou rénovés par le Projet "Santé - Nyong et Mfoumou" qui a financé l'achat du matériel nécessaire aux consultations, aux soins infirmiers et à toutes les activités d'un centre de santé. Le stock initial de médicaments et une moto entraient également dans le financement du Projet qui a duré quatre ans, de 1990 à 1994. Les effectifs des personnels affectés dans les centres de santé, qui ne correspondaient pas toujours aux normes que le projet s'était fixées, variaient de une à quatre personnes, certains centres étaient dirigés par des aides-soignants. L'hôpital de district d'Endom - devenu centre médical d'arrondissement par la suite - n'était pas concerné par le projet. C'est ainsi que les trois centres de santé de l'arrondissement ont été rénovés, les médicaments et le matériel technique y étaient disponibles, et excepté ce qui concerne les ressources humaines, leur plateau technique était supérieur à celui de la structure de référence ; les médicaments et le matériel étaient dans les centres de santé, les personnels qualifiés, à l'hôpital de référence.

21 Le coordinateur des soins de santé primaires de cette époque raconte la situation suivante : "Je suis parti en supervision au centre de santé d'Akak. J'ai trouvé un malade que l'infirmier chef avait décidé de référer à l'hôpital depuis deux jours. On ne trouvait pas de véhicule pour l'amener à Endom, je l'ai ramené à la fin de la supervision. Le médecin d'Endom a tout de suite examiné le malade et prescrit des médicaments en le mettant en observation immédiate. L'hôpital d'arrondissement ne disposait d'aucun médicament et la pharmacie de la ville était vide. La famille a loué une moto pour aller acheter les médicaments dans un autre centre de santé à douze kilomètres (Ekoudou)"6. Dans ce cas les malades préfèrent aller directement à l'hôpital protestant de Metet qui appartient à un autre district mais dont les soins sont jugés de meilleure qualité. De plus, pour des raisons sociales et financières, les familles des malades et les malades préfèrent se rendre dans un hôpital où ils ont des connaissances parmi le personnel; un malade nous confiait : "Ils m'ont envoyé à Akonolinga quand la maladie les a dépassés. Moi, je n'ai personne là-bas, je ne connais personne. Alors je suis venu à Metet, ici, on te soigne toujours, on demandera l'argent après et il y a la radio pour voir la maladie".

De plus, les personnels de santé ne prennent pas la peine de remplir les fiches de référence, et la structure de référence qui reçoit le malade référé n'adresse aucune 
rétro-information au centre de santé initial. Les personnels justifient leur comportement par un excès de travail. Or la charge de travail des centres de santé du district est de 2,3 consultations par jour. Par ailleurs nombre de malades qui s'adressent à l'hôpital de référence ne sont pas enregistrés dans les documents officiels de la formation sanitaire, car, ils ont payé ce qu'on appelle le "K direct", c'est-à-dire le paiement d'un acte directement au médecin et non à la caisse de l'hôpital. Le rapport d'activités du district d'Endom de 1993 révèle que tous les centres de santé disposent d'un cahier pour consigner les malades référés, mais deux seulement l'ont utilisé plus de cinq fois au cours de l'année; et sur un total de 33 malades enregistrés comme référés, les centres n'ont reçu que deux fois une rétro-information écrite.

La participation communautaire

23 La politique de santé du Cameroun a créé des structures de représentation de la communauté appelées Comités de santé (COSA). Deux missions sont confiées à ces comités : participer à la gestion financière des structures de santé et organiser la communauté pour la mobiliser en vue d'actions favorables à la santé. Les comités de santé élisent en leur sein une structure spécialisée appelée Comité de Gestion (COGE) qui assume la première mission. La mobilisation sociale incombe au COSA. Mais la mission des COSA n'a pas été clairement définie au début de la réforme ${ }^{7}$. Au départ les membres du COSA devaient informer les populations de ce qui se fait et se dit au centre de santé, et porter à l'attention de l'équipe technique du centre les doléances des communautés. Les membres des COSA devaient également participer au recensement de la population de l'aire de santé avant le démarrage des activités dans l'aire de santé. Mais après l'engouement des débuts, les COSA sont rapidement devenus inactifs. Nous l'avons dit, ses tâches n'étaient pas clairement définies; de plus les personnes désignées n'étaient pas prêtes à se dévouer pour leur communauté, leur niveau de formation ne leur permettant pas d'assumer les responsabilités que les concitoyens attendaient d'eux, et les personnels de santé s'accommodaient difficilement d'un partage du pouvoir de gestion relevant de leur domaine.

Lorsqu'il s'est agi d'élire les membres des COSA, l'ensemble des partenaires impliqués dans le projet de mise en place du district étaient contraints par le temps. La sensibilisation se faisait très rapidement et de manière superficielle pour créer des comités de santé, quelles que soient les personnes désignées. Certains ont supposé que l'élection au sein du comité de santé procurerait des avantages : rémunération, gratuité des soins pour la famille, etc. Des notables villageois ont brigué les places dans les COSA, mais sans intention réelle de représenter la communauté, ni même de se rendre disponible. Or, contrairement aux COGE qui tiennent leurs réunions tous les mois, celles du COSA ne sont prévues que deux fois par an, et les membres du COGE perçoivent annuellement quatorze indemnités au titre des réunions, ceux du comité de santé, seulement deux. Les soignants qui étaient supposés mettre en place ces COSA et leurs activités n'étaient pas formés pour cette tâche; les séminaires sur les soins de santé primaires auxquels ils avaient assisté ne les avait pas suffisamment apprêtés à jouer ce rôle.

En ce qui concerne le fonctionnement des comités de gestion, le Projet Santé Nyong et Mfoumou avait clairement décrit les activités qui permettaient à leurs membres de participer à la gestion financière des centres de santé. Les recettes des centres produites par le recouvrement de coûts (ventes des médicaments, consultations, soins, adhésions) sont placées sur un compte d'épargne postale. Pour effectuer un retrait, 
deux conditions sont requises : le trésorier et le président du comité de gestion de l'aire de santé doivent signer, et le chef de service de santé d'arrondissement doit avaliser la dépense. Le commis conserve l'argent qu'il a perçu dans une petite caisse, et le trésorier du comité de gestion récupère cet argent en fin de semaine pour le placer dans le coffre du centre; ce coffre est muni de deux cadenas et ne peut s'ouvrir qu'en la présence du trésorier et du commis, chacun détenant une clef. Le coffre ne doit jamais contenir plus de $500 \mathrm{FF}$, au-dessus, l'argent doit être versé dans le compte d'épargne.

Tous les centres de santé ont connu des détournements et des malversations dans la gestion des fonds ainsi gardés. Les professionnels de santé usent de leur pouvoir et réussissent à obtenir "un prêt" personnel avec l'accord du comité de gestion ou au moins de son président, du trésorier et du commis, prêt qui sera évidemment très difficile, voire impossible à recouvrer. Des médicaments "disparaissent" dans la pharmacie tenue par le commis, ce qui représente un manque à gagner pour le centre mais pas pour le commis ou les autres membres du comité de gestion. À moins que le président, le trésorier et le commis du comité de gestion s'entendent pour faire disparaître de l'argent au moment du transfert de la caisse du centre vers le compte d'épargne. Ces détournements peuvent parfois atteindre des sommes importantes, surtout en cas de non respect des mesures de gestion prescrites, qui peuvent amener le commis à disposer de la recette de trois semaines d'activités du centre avec des sommes pouvant aller jusqu'à $300.000 \mathrm{FCFA}^{8}$. Cette situation révèle aussi un manque de supervision et de contrôle de la part du district.

Le statut juridique des comités de santé a tardé à être précisé. Nés dans le sillage des lois sur la liberté d'association de 1990, souvent ces comités ne sont pas formellement déclarés à l'autorité administrative et n'ont pas de statut propre. Depuis 1995, le décret présidentiel qui crée les districts de santé stipule que les comités de santé et de gestion font partie de ces districts, mais sans préciser leurs fonctions ni leur statut juridique. Aussi, les poursuites judiciaires pour détournements de fonds sont impossibles, il s'ensuit une totale impunité pour les personnes indélicates, ce qui n'est évidemment pas dissuasif pour les membres à venir de ces comités.

La collaboration intersectorielle

28 La collaboration intersectorielle préconisée par la réforme se résume le plus souvent à des expériences ponctuelles qui dépendent davantage des hommes en poste à un moment donné que d'une organisation institutionnelle. La structure du district de santé d'Akonolinga, divisé en trois unités administratives rend difficile cette collaboration intersectorielle, puisque le district est alors censé collaborer avec les trois comités de développement de ces trois unités administratives et qui ont parfois des intérêts divergents. Cette collaboration a pu se traduire, par exemple, pour le district d'Akonolinga, par une demande d'une école, d'un collège, d'une association de jeunes ou de femmes aux responsables sanitaires du district de santé d'organiser une conférence dans le cadre de leurs activités sur un sujet sanitaire qui les intéressent, le plus souvent le sida et les maladies sexuellement transmissibles. Il arrive aussi que pour une activité précise, par exemple l'organisation des journées nationales de vaccination, les responsables de la santé du district demandent et obtiennent des autres secteurs une aide logistique, en l'occurrence le prêt de véhicules.

Une expérience qui s'est déroulée dans l'arrondissement d'Endom illustrera les résultats possibles d'une collaboration intersectorielle, mais également les obstacles et les enjeux d'une telle démarche. Au début de l'année 1992, l'agglomération urbaine 
d'Endom n'est plus approvisionnée en eau potable: les installations du système qui alimentaient la ville en eau potable ne fonctionnaient plus car la Société nationale d'électricité du Cameroun avait coupé l'électricité en raison du non paiement des factures. La population se débrouillait alors comme elle pouvait, en allant s'approvisionner en eau potable dans un autre centre à quatre kilomètres de la ville, et en utilisant l'eau des puits et des marigots. Le délégué de l'agriculture ${ }^{9}$ supervisait alors un projet de pépinière de plans de palmier à huile qui nécessitait beaucoup d'eau. Pour sauver sa pépinière, après avoir été encouragé par les autorités administratives, il évalue les ressources et les travaux nécessaires pour faire fonctionner les installations et une première phase de la réhabilitation est financée entièrement par le service d'arrondissement d'agriculture et imputée au projet de la pépinière. Deux semaines plus tard, la ville entière bénéficiait à nouveau de l'eau potable. A la fin du projet de pépinière, le sous-préfet, sur l'initiative du délégué à l'agriculture, du chef de service de santé d'arrondissement et du percepteur de la recette des finances, convoque une réunion de tous les responsables des services publics de l'arrondissement ; l'objectif de cette réunion est de convaincre les autorités locales qu'il est possible de faire fonctionner le système d'approvisionnement en eau avec des moyens réduits et si chacun contribue : le médecin fait un exposé sur l'importance de l'eau dans la vie de la communauté, le délégué de l'agriculture a présenté le projet qui s'inspirait de l'expérience qu'il venait de conduire, et le percepteur promet des facilités de paiement aux chefs de services qui participeraient à l'opération ; décision est alors prise d'organiser un recouvrement mensuel auprès de tous les fonctionnaires de la ville pour financer le fonctionnement du système d'approvisionnement en eau potable. Le projet fonctionna normalement pendant trois mois et un agent de la délégation de l'agriculture, originaire de l'arrondissement, fut désigné pour être formé à la maintenance des machines. La troisième phase du projet devait consister à élargir la demande à tous les utilisateurs, et la population fut sollicitée pour apporter une contribution mensuelle. La majorité de la population était d'accord, d'autant plus que la somme à verser était peu élevée, et le souvenir des trois mois passés sans eau en début d'année restait présent dans les esprits. Le proviseur du lycée adressa un message à tous les parents d'élève pour expliquer les bienfaits de l'opération. Mais l'approche d'échéances électorales fut préjudiciable à cette initiative. Les élites ${ }^{10}$, en période électorale, se souviennent alors de leur arrondissement d'origine. Le comité de développement ressuscita et fut convoqué d'urgence; et le sous préfet prit l'initiative de présenter le projet de financement de l'approvisionnement en eau aux élites. Ces dernières informent alors séance tenante la population et les fonctionnaires qu'ils n'auront plus rien à payer et qu'elles s'occuperaient du problème dès la semaine suivante. Un proche de ces élites, originaire de la communauté, fut chargé de se rendre à Yaoundé pour percevoir la somme de 300.000 FCFA nécessaire à l'achat du gasoil permettant de faire fonctionner les installations pour le mois en cours. L'argent n'est jamais arrivé à Endom, ni pour le mois en cours, ni pour les mois suivants, et le projet s'arrêta.

Les ressources humaines et le financement

Le district se trouve en dessous des normes définies par la REOSSP, avec de plus une concentration du personnel dans les formations sanitaires en zone urbaine. En effet, on compte quatre médecins généralistes, pour 77.359 habitants soit, un médecin pour 19.384 habitants, si nous intégrons en plus le fait que ces médecins habitent dans les deux villes du district où réside $40 \%$ de la population, le district est en-deçà de la 
moyenne nationale qui s'établit à un médecin pour $13.902^{11}$. De plus le cadre conceptuel d'un district de santé viable prévoit que "Au sommet du système de santé de district se trouve le bureau de santé de district dirigé par une équipe multidisciplinaire supervisée par un médecin de santé publique"12, ce qui n'est pas le cas ici.

Tableau 2: Personnels des services publics de santé

\begin{tabular}{|c|c|c|c|c|c|c|c|}
\hline \multirow[t]{2}{*}{ Personnels } & \multicolumn{7}{|l|}{ Etablissements } \\
\hline & \begin{tabular}{|ll} 
Service & de \\
santé & de \\
district &
\end{tabular} & $\begin{array}{l}\text { Hôpital de } \\
\text { district }\end{array}$ & $\begin{array}{l}\text { Centre } \\
\text { d'arrondissement } \\
\text { d'Endom }\end{array}$ & médical & $\begin{array}{l}\text { Ensemble } \\
\text { centres d } \\
\text { intégrés }\end{array}$ & $\begin{array}{r}\text { des } \\
\text { de santé }\end{array}$ & $\begin{array}{lll}\text { setal } & \\
& \end{array}$ \\
\hline Médecins & 1 & & & 3 & 1 & & 4 \\
\hline $\begin{array}{l}\text { Techniciens } \\
\text { supérieurs de soins } \\
\text { infirmiers }\end{array}$ & 0 & & & 1 & 0 & & 1 \\
\hline $\begin{array}{l}\text { Techniciens médico- } \\
\text { sanitaires }\end{array}$ & 1 & & & 1 & 0 & & 3 \\
\hline $\begin{array}{l}\text { Infirmiers diplômés } \\
\text { d'État }\end{array}$ & 1 & & & 3 & 0 & & 10 \\
\hline $\begin{array}{l}\text { Infirmiers brevetés et } \\
\text { agents techniques }\end{array}$ & 2 & & & 6 & 2 & & 12 \\
\hline $\begin{array}{l}\text { Infirmiers brevetés } \\
\text { accoucheurs }\end{array}$ & 1 & & & 3 & 0 & & 4 \\
\hline $\begin{array}{l}\text { Techniciens adjoints } \\
\text { de laboratoire }\end{array}$ & 0 & & & 6 & 0 & & 9 \\
\hline Aides-soignants & 2 & & & 11 & 4 & & 26 \\
\hline Agents de l'État & 2 & & & 7 & 3 & & 14 \\
\hline Total & 11 & & & 41 & 10 & 23 & 85 \\
\hline
\end{tabular}

La situation est encore plus critique pour les centres de santé. La norme pour la mise en place d'un paquet minimum de prestations pour une population de 5.000 à 10.000 personnes est : un infirmier diplômé d'État, un infirmier accoucheur et un aidesoignant, soit un effectif minimum de trois personnes par centre de santé. Or pour l'ensemble des 10 centres de santé on ne dénombre que 23 personnes, et un centre de santé est dirigé par un aide-soignant. L'insuffisance de personnels est à relier à la politique de réduction des effectifs dans la fonction publique. Par ailleurs, ce personnel 
déjà insuffisant est démotivé comme en témoigne cette infirmière de l'hôpital de district d'Akonolinga.

"Je suis infirmière depuis 1986, avant, je gagnais bien ma vie, j'envisageais de repartir à l'école pour devenir infirmier diplômé d'État. Maintenant, je gagne moins que la bourse que l'État me donnait quand j'étais à l'école et je ne peux pas rentrer à l'école, il paraît que c'est interdit. Le salaire ne nourrit pas les enfants. A l'hôpital, tu vois toujours des malades, tu ne peux rien faire pour eux, ils n'ont pas d'argent et il n'y a pas le matériel, alors, je fais ma part de soins à la maison pour nourrir mes enfants. Même le gouvernement oublie les gens qui soignent; on a dit que l'argent des policiers ne baisse pas, on a donné des primes aux percepteurs, aux enseignants, aux magistrats, et nous? rien! Alors chacun se débrouille comme il peut".

La démotivation du personnel dépend autant de la baisse des salaires, du gel des avancements, des conséquences de la dévaluation que des conditions de travail. De plus, le manque de reconnaissance du travail accompli complète cette démotivation.

Les districts de santé d'Akonolinga et d'Endom ont d'abord bénéficié de trois sources de financement: public (infrastructures, salaires des agents, et accessoirement fonctionnement), de la coopération bilatérale et d'organismes privés (AFVP, secteur privé confessionnel, la Société de développement du cacao du Nyong et Mfoumou), et communautaire.

Les mécanismes d'utilisation de l'allocation budgétaire publique passent par plusieurs médiateurs des finances, ce qui rend difficile l'utilisation de ce crédit à $100 \%$. Il est reconnu que $30 \%$ vont passer en pots-de-vin et surfacturation; il faut savoir également que le taux d'exécution du budget de l'État dépasse rarement $80 \%$ ces dernières années, il devient alors difficile de savoir quelle sera la contribution du budget national avant la fin de l'exercice. Le plan d'action du district doit donc être élaboré en début d'exercice, et le district doit gérer cette incertitude tout au long de l'année. Aussi, pendant la durée du projet AFVP, le financement public pour la mise en place des districts n'était pas pris en compte, l'utilisation du budget public étant laissée à l'appréciation du responsable de la structure de soins.

35 La communauté participe au financement par trois mécanismes d'importance inégale. $\mathrm{Au}$ début du projet, chaque famille devait payer un droit d'adhésion au projet (cette participation a largement permis la rénovation des centres de santé), les membres de la communauté devaient ensuite participer manuellement aux travaux de construction ou de rénovation, enfin de manière continue la communauté participe en achetant les médicaments, en payant les consultations et les soins. Cette dernière source de financement, outre les détournements mentionnés, doit aussi faire face à la concurrence d'un réseau parallèle de vente de médicaments et de prestations de soins illégales à but lucratif.

Avec la fin du projet AFVP, les financements se sont arrêtés brutalement, le district s'est retrouvé avec un véhicule usagé à entretenir, et des motos dont l'amortissement n'avaient pas été pris en compte. La supervision n'était plus assurée. Les détournements étaient plus fréquents et les médicaments disparaissaient de plus en plus. Le dépôt départemental de médicaments essentiels mis en place pour faciliter le ravitaillement des centres de santé a disparu. Certains centres ont purement et simplement fermés, d'autres ont retrouvé leur situation d'avant le démarrage du projet. 


\section{NOTES}

1. Endom est un arrondissement limitrophe de celui d'Akonolinga. Cet arrondissement constituait un district de santé à part entière avant le séminaire de Bertoua qui a décidé de l'assimiler au district d'Akonolinga. La population d'Endom (19.000 habitants) était jugée insuffisante pour un district de santé viable.

2. La population d'un village limitrophe de cette aire de santé avait refusé de faire partie de cette aire de santé parce qu'elle estimait qu'elle n'avait aucun rapport avec les autres communautés intégrées à l'aire de santé.

3. Lorsque le Président de la république crée un arrondissement, il désigne en même tant son chef lieu. Le village choisi bénéficiera des investissements publics et du rapprochement avec les services administratifs.

4. Il s'agit de volontaires de l'Association française des volontaires du progrès (AFVP).

5. Propos rapporté par un membre du projet AFVP dans le rapport d'une réunion de concertation tenue à Edjom en 1991.

6. Entretien avec l'ancien coordinateur des soins de santé primaires de l'arrondissement d'Endom, avril 1999.

7. Ce n'est qu'en 1998 qu'un document a été élaboré par le ministère de la santé pour décrire ce que le système de santé doit attendre des comités de santé, c'est-à-dire la mobilisation des populations pour l'organisation des initiatives communautaires en faveur de la santé.

8. Un procès verbal d'une réunion tenue au centre de santé d'Edjom en août 1993 mentionne un achat de médicaments hors du dépôt départemental pour un montant de 350.000 FCFA. Cet argent avait été remis directement à l'infirmier chef pour acheter les médicaments à Yaoundé, ce qui est contraire à toute la procédure.

9. Responsable des service d'agriculture dans l'arrondissement. Il est chargé du développement agricole.

10. C'est-à-dire toutes les personnes influentes originaires de la communauté, résidant sur place ou, et c'est le cas la plupart du temps, en fonction dans une autre ville. L'arrondissement d'Endom regorge d'élites politiques très influentes au niveau national.

11. Document DEPI/ MSP (1998), repris dans le Plan national de développement sanitaire.

12. Cadre conceptuel d'un district de santé viable au Cameroun. Ministère de la Santé / DEPI / Banque mondiale, Yaoundé, 1999.

\section{AUTEUR}

\section{RAPHAËL OKALLA}

Service de coopération et d'action culturelle, BP 2105 Libreville Gabon, e-mail : rokalla@iccnet.cm. 